\title{
An overview about erythrocyte membrane
}

\author{
Sofia de Oliveira* and Carlota Saldanha \\ 1 Unidade de Biologia Microvascular e Inflamação, Instituto de Medicina Molecular, Instituto de \\ Bioquímica, Faculdade de Medicina, Universidade de Lisboa, Lisboa, Portugal
}

Received 29 May 2009

Accepted 7 September 2009

\begin{abstract}
In the sixties and seventies, erythrocytes or red blood cells (RBCs) were extensively studied. Much has been learnt particularly concerning their metabolism and gas transporter function.

In the past decade, the use of new approaches and methodologies, such as proteomic analysis, has contributed for a renewed interest on the erythrocyte. Recent studies have provided us with a more detailed and comprehensive picture on the composition and organization of its cellular membrane that will be the main subject of this minireview. Unexpectedly, it has been recognized that this cell expresses several adhesion molecules on its surface, like other cellular types such blood circulating cells or endothelial cells. Taking into consideration the cellular functions of the erythrocyte, the clarification of the role of those adhesion molecules may in the future open new horizons for the biological significance of this cellular player.
\end{abstract}

Keywords: Erythrocyte, erythrocyte membrane, membrane structure and adhesion molecules

\section{Introduction}

Erythrocytes are the major cell component of blood. These red cells are a product of a differentiation process that starts in the bone marrow where hematopoietic stem cells differentiate to nucleate RBCs. After extrusion of nuclei and degradation of endoplasmic reticulum, reticulocytes emerge in the circulation; here they rapidly develop into mature RBCs $8 \mu \mathrm{m}$ biconcave disk and with 120 days life span [36]. Despite these features, the protein and lipid composition of the RBCs is subject to change during its life-time. This can be particularly observed at the level of its plasma membrane.

The RBC membrane content is similar with most of animal membranes and it is composed by: $19.5 \%$ $(\mathrm{w} / \mathrm{w})$ of water, $39.5 \%$ of proteins, $35.1 \%$ of lipids and $5.8 \%$ of carbohydrates [52].

Interestingly during several years scientist believe that RBC membrane was composed just by a punch of lipids and one single protein. In the 20th century, during the 1960s and 1970s, several methods were developed to analyze red cell membrane content in normal RBCs. Amazingly, the first studies using a sodium dodecyl sulphate polyacrylamide gel electrophoresis (SDS-PAGE) reveal that red cell membrane ghosts had more than just one single protein, indeed it became clear that RBC membrane composition just started to be uncovered. Nowadays Pasini et al. had already made the identification of at least 340 different red cell membrane proteins [36].

Haemoglobin is the major protein in RBCs, and it gives to red cells the ability to transport oxygen and carbon dioxide to and from tissues, for this reason the membrane of RBCs is extremely important

\footnotetext{
${ }^{*}$ Corresponding author: Sofia de Oliveira, Microvascular Biology and Inflammation Unit, Institute of Molecular Medicine, Av. Professor Egas Moniz, 1649-028 Lisbon, Portugal. Fax: +351 217999 477; E-mail: sloliveira@fm.ul.pt.
} 
for them. This highly and specialized function of transport oxygen and carbon dioxide, is known to be the main one of red cells in blood, however there are some others also very important such as nitric oxide transportation and signalling. Surprisingly several recent studies $[16,27,45,46]$ reveal that RBCs have also the ability to express numerous adhesion molecules. Additionally RBCs are being recognized to have other functions, that 40 years ago were unthinkable, such as the transport of $\mathrm{iC} 3 \mathrm{~b} / \mathrm{C} 3 \mathrm{~b}$-carrying immune complexes [36] or even an influencing factor on leukocytes and platelets margination and adhesion due to one of the most studied features of red cells, aggregation [33]. Concerning RBCs main functions any abnormality that occurs in red cell membrane could be a problem or even fatal.

The first disorder exhaustively studied was hereditary elliptocytosis (HE) after that several more disorders were being identified until our days. There are red cell protein disorders and also red cell lipid disorders. However the lipid disorders appear to be more atypical than the protein ones. Yawata et al. [52] had detected a significant number of patients with hereditary high red cell membrane phosphatidylcholine haemolytic anemia (HPCHA), the most common lipid disorder known until now.

This minireview will be focus on the RBCs membrane structure, composition and organization.

\section{RBC membrane lipids}

The RBC membrane is composed by $60 \%$ of phospholipids, essentially phosphatidylcholine (PC), phosphatidylethanolamine (PE), sphingomyelin (SM) and phosphatidylserine (PS). It has also some phospholipidic minor components such as phosphatidylinositol (PI), PI-monophosphate (PIP), PI4,5-bisphosphate $\left(\mathrm{PIP}_{2}\right)$, phosphatidic acid (PA), lysophosphatidylcholine (Lyso-PC) and lysophosphatidylethanolamine (Lyso-PE). Non-sterified cholesterol represents about 30\% of the lipidic RBC composition, and the last $10 \%$ are glycolipids [52]. At the physiologic $\mathrm{pH}$, the majority of phospholipid content is electrically neutral, although PS, PA and PI are negatively charged. With the exception of SM and lyso-PC, the bulk of phospholipids have two fatty acid chains attached to a glycerol backbone. The most common fatty acids in human lipid bilayer membranes are usually the following: (16:0, 18:0, 18:1, 18:2 and 20:4). Concerning the glycolipid content, it is mainly based on sphingosine, such as glycosphingolipids. It is known that the sugar residues present in glycolipids are responsible for numerous functions such as adhesiveness to the extracellular space. Those reasons explain the location, almost exclusively, of glycolipids to the outer leaflet of the bilayer.

In all eukaryotic membranes lipids are asymmetrically distributed across the bilayer plan, this is known as trans asymmetry. This asymmetric distribution has a very important structural and functional role. In human RBCs, PS and PE are located almost entirely in the inner monolayer while PC and SM are more common in the outer leaflet. An exposure of PS in the outer surface leads to several mechanisms to achieve cell death, apoptosis [52]. This lipid asymmetry reflects a steady state that involves a constant exchange of phospholipids by a flip/flop mechanism between the two lipid leaflets. The transmembrane shuttle of these phospholipids in biological membranes is extremely fast. Interestingly membrane phospholipids asymmetry results from a balance between an active translocation of PS and PE and a passive slow bidirectional flip/flop movement of phospholipids [52]. The trans asymmetry is produced and maintained by an adenosine triphosphate (ATP) dependent transport system. Two important enzymes are responsible for the translocation of phospholipids: flippase is responsible for translocate PS and PE from the outer to the inner leaflet, floppase catalyzes the translocation of the other lipids from the inner to the outer leaflet of the bilayer. Additionally flippase is recognized as a member of the $\mathrm{Mg}^{2+}$ dependent enzyme and P-glycoprotein ATPases family whereas floppase is a multidrug resistance protein 1 (MRP1) family member. 
As mature RBC are not able to synthesize lipids de novo, the repair and renewal of phospholipids is dependent on several mechanisms, for instance lipid exchange and acylation of fatty acids with plasma lipoproteins [52].

Red cell deformability is one of the most known and unique characteristics of RBC [2,47]. The RBC shape is ultimately determined by membrane proteins, especially spectrin network, and also by the lipid bilayer content [52]. RBC are able to maintain the its discoid shape, and yet allowing cytoskeleton rearrangements that permit it to pass through capillaries, and then restore again its normal shape without cell fragmentation [36,52]. Considerably lipids are crucial in the maintenance of RBC shape. Minimal changes in the surface area (inner or outer) could be liable for several abnormalities either in morphology or function as will discussed later on in RBC disorders topic.

Red cell membrane fluidity is also a very important and crucial point for the red cell functions. Membrane lipid fluidity is in general dependent on several factors, such as (a) the class of phospholipids, (b) the degree of saturation of the fatty acids, (c) the length of the acyl chains, (d) the type of cholesterol (free or esterefied) and also (e) the presence or absence of amphipathic compounds such as lysophosphatides. For example, as the degree of desaturation of fatty acids increases, the packing of hydrophobic tails in the core of the bilayer is increasingly disrupted, thereby enhancing the membrane fluidity. Interestingly fatty acid composition in mammalian erythrocytes has also an enormous influence on cellular aggregation, besides other factors of course [38].

A last point concerning lipids will be the specific membrane domains, usually called as lipid rafts. This subject will be discussed following.

\section{Lipid-rich microdomains/Lipid rafts}

Lipid rafts are lipid-rich microdomains that are characterized by having a low density and being insoluble in cold nonionic detergents. They have several acronyms such as detergent-resistant membranes (DRM), Triton-insoluble membranes (TIM) and triton-insoluble floating fractions (TIFF) [37].

Lipid rafts have a high level of organization. As they are enriched in cholesterol and glycosphingolipids (such as gangliosides (Gang) and sulfatides) with saturated fatty acylchains they possess a lower fluidity than bulk plasma membrane that contains less cholesterol, SM and Gang, and more phospholipids with unsaturated acyl chains [30,37,52].

These microdomains contain over $4 \%$ of total RBC membrane protein [19]. The major integral proteins in RBC rafts are stomatin, flotillin-1 and flotillin-2 [42,52]. Duffy protein receptor and glycosylated phosphatidylinositol-anchored proteins (GPI-proteins: CD55, CD58, CD59) are also characteristic of lipid rafts [37].

Accordingly to a more conservative interpretation, lipid rafts are probably structures with an average diameter in the range of 100-200 nm [30]. Still different methodological approaches may yield different interpretations. There are specialized lipid rafts with different protein or lipid composition. For instance, a subclass of lipid rafts, caveolae, are specifically enrich in protein caveolin. Importantly, these lipid rafts have been implicated in specific signalling functions.

During this last decade several studies point out to a very important role, or even a key role of red cell lipid rafts in regulating parasite infections, such as in the malarial infection $[32,43]$.

\section{RBC membrane proteins}

Red cell membrane proteins are classified into two groups according to the easiness with which they can be separated from membranes. Some are easily isolated by high- or low-salt or high-pH extraction, 


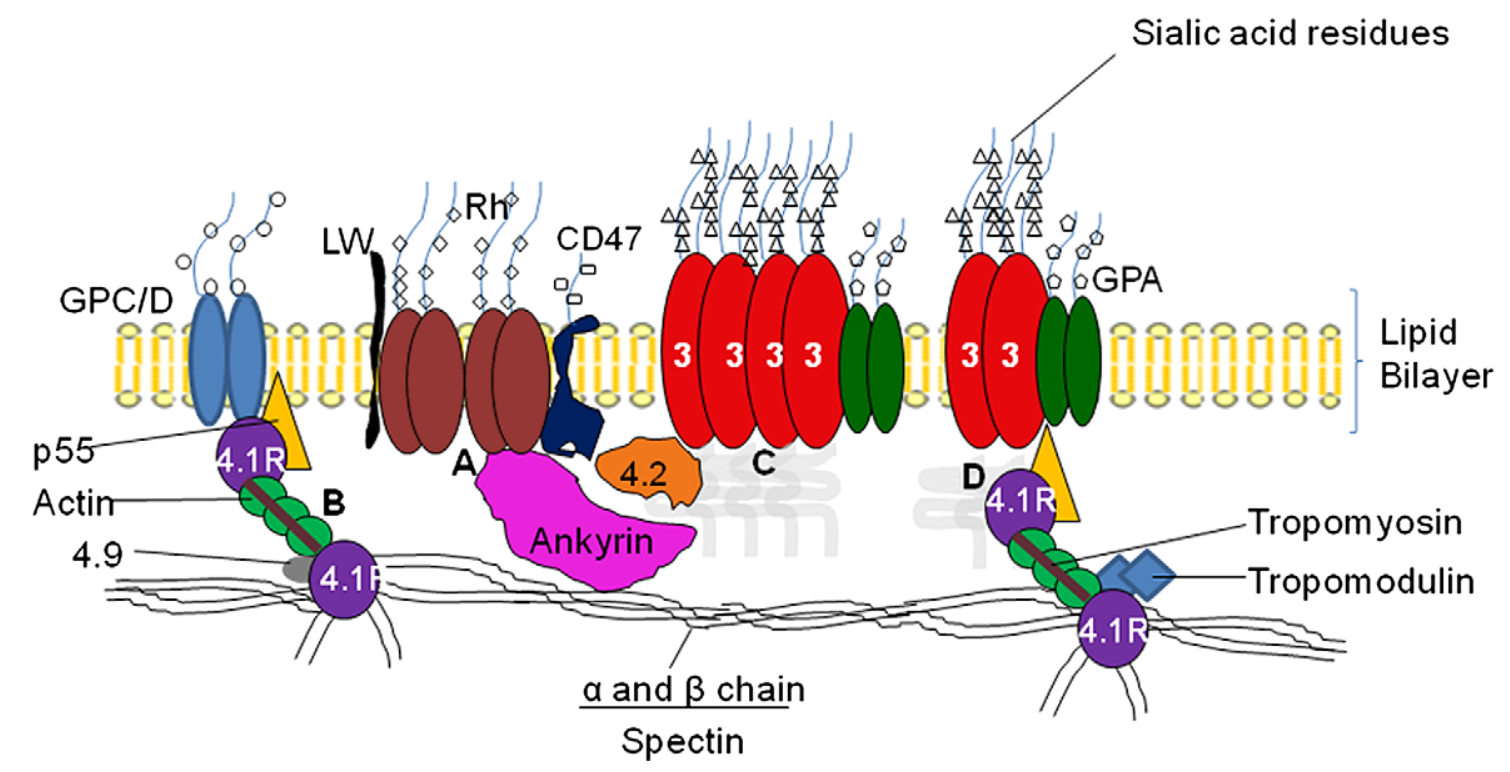

Fig. 1. Simplified diagram of the RBC membrane structure. (A) Rh complex; (B) protein 4.1 complex; (C) and (D) band 3 macrocomplex ((C) band 3 tetrameric form and (D) band 3 dimeric form) [3].

perhaps because they appear to be connected with only one face of the membrane. This group of proteins is commonly called as the "peripheral proteins", such as spectrins. A second group, the "integral proteins", can be extracted only by harsh reagents, frequently detergents, since they are strongly embedded into or through the lipid bilayer. Band 3 and glycophorins are the most representative integral proteins (see Fig. 1).

Additionally, erythrocyte membrane proteins can also be categorized in terms of protein function into three groups (a) cytoskeletal proteins (spectrin, actin, protein 4.1, etc.), (b) integral structural proteins (band 3, glycophorins, etc.) and (c) anchoring proteins (ankyrin, protein 4.2, etc.).

In the further topics the structural and functional aspects of the most important proteins (see Table 1) of red cell membrane will be discussed generally.

\section{Integral proteins}

\subsection{Band 3}

Protein band 3 or anion exchanger (AE1) is the major integral protein present in RBC membrane ( $10^{6}$ copies per cell). It is a 911 amino acid multispanning membrane protein with molecular weight of $100 \mathrm{kDa}$, with 12-14 transmembrane (TM) segments [39].

Band 3 has an N-terminal cytoplasmic domain (amino acid 1-359) that can be associated to several proteins. This domain is responsible for the main membrane anchorage site of cytoskeleton. Therefore it is very important for the flexibility and rigidity of RBC. The N-terminal domain is the site for binding of ankyrin, proteins 4.2 and 4.1, and also glycolytic enzymes (GEs) and haemoglobin [4,8,9,31,35,39, 41,52,53,55]. The band 3 C-terminal domain (amino acid 360-911) carries out the anion exchange. It is formed by 12-14 TM segments with a short cytoplasmic tail (33 amino acids). Additionally it is responsible for the binding of carbonic anhydrase II [4,8,9,31,35,39,41,51,53,55]. 
Table 1

Genetic characteristics of the most famous red cell membrane protein

\begin{tabular}{lcccc}
\hline Protein & Gene symbol & Chromosome location & Amino acids & Related diseases \\
\hline$\alpha$-spectrin & SPTA1 & $1 \mathrm{q} 22-\mathrm{q} 23$ & 2429 & HE, HPP, HS \\
$\beta$-spectrin & SPTB & $14 \mathrm{q} 23-\mathrm{q} 24.2$ & 2137 & HE, HPP, HS \\
Ankyrin & ANK1 & $8 \mathrm{p} 11.2$ & 1881 & HS \\
$\alpha$-adducin & ADDA & $4 \mathrm{p} 16.3$ & 737 & - \\
$\beta$-adducin & ADDB & $2 \mathrm{p} 13-\mathrm{p} 14$ & 726 & - \\
Band 3 (AE1) & EPB3 (SLC 4A1) & $17 \mathrm{q} 21-\mathrm{q} 22$ & 911 & HS, SAO, RTA \\
Protein 4.1 & EL1 (EPB41) & $1 \mathrm{p} 36.1$ & 588 & HE \\
Protein 4.2 & ELB42 & $15 \mathrm{q} 15$ & 691 & - \\
p55 & MPP1 & Xq28 & 466 & - \\
$\beta$-actin & ACTB & $7 \mathrm{p} 12-\mathrm{p} 22$ & 375 & - \\
Tropomodulin & TMOD & $9 \mathrm{q} 22.2-\mathrm{q} 22.3$ & 359 & - \\
G3PD & GAPD & $12 \mathrm{p} 13.1-\mathrm{p} 13.31$ & 335 & - \\
Stomatin & EPB72 & $9 \mathrm{q} 34.1$ & 288 & - \\
Tropomyosin & TPM3 & $1 \mathrm{q} 31$ & 239 & - \\
Glycophorin A & GYPA & $4 \mathrm{q} 28.2-\mathrm{q} 31.1$ & 131 & - \\
Glycophorin B & GYPB & $4 \mathrm{q} 28.2-\mathrm{q} 31.1$ & 12 & HE \\
Glycophorin C & GYPC & $2 \mathrm{q} 14-\mathrm{q} 21$ & 107 & HE \\
Glycophorin D & GYPD & $2 \mathrm{q} 14-\mathrm{q} 21$ & 59 & - \\
Glycophorin E & GYPE & $4 \mathrm{q} 28.2-\mathrm{q} 31.1$ & & -
\end{tabular}

Notes: HE - hereditary elliptocytosis; HPP - hereditary pyropoikilocytosis; HS - hereditary spherocytosis; SAO - Southeast Asian ovalocytosis; RTA - renal tubular acidosis.

Although band 3 exists in RBC membrane as dimers (70\%) it can also be present as tetramers or higher order oligomers (30\%) (Fig. 1). The tetrameric form binds basically protein 4.2 and ankyrin [38]. Altogether, B3 and respective associated proteins are known as the B3 macrocomplex. B3 macrocomplex is also related with glycophorin $\mathrm{A}$ and RhAG and/or Rh complex [51].

Like several other structural proteins, band 3 configuration can also be modulated by phosphorylation - via phosphotyrosine kinases (PTKs) like p53/56 ${ }^{\mathrm{Lyn}}$ - or dephosphorylation - via phosphotyrosine phosphatase (PTP). These biochemical reactions are responsible for instance for the release of GEs to the cytoplasm and their subsequent activation $[3,9,35,41,56]$.

\subsection{Glycophorins: Glycophorin A (GPA)}

Sialic acid residues are abundant in negatively charged RBC surface. This negative net charge is mostly conferred by the sialic residues (60\%) present in Glycophorin A (GPA) (Fig. 1), but also in other glycophorins (GPs), band 3 and some glycolipids. The negative surface charge of RBC plays a crucial role in modulating RBC-RBC interactions and as well $\mathrm{RBC}$ interactions with vascular endothelium and the other circulating blood cells $[46,52]$.

Glycophorins or syaloglycoproteins are about $2 \%$ of total RBC membrane protein [52]. Among them GPA is the major constituent representing $1.6 \%$ of total RBC membrane protein. Others such as GPB/E, similar to GPA, and GPC/D are present in lower amount. Interestingly GPC/D are non erythroid-specific.

Glycophorins have three domains, (1) a cytoplasmic domain, which contains a cluster of basic residues that are located near the plasma membrane, (2) a hydrophobic domain which exists as a single $\alpha$-helix spanning the lipid bilayer and (3) a extracellular domain which is heavily glycosilated [52]. 
GPA is a 131 amino acid syaloglycoprotein with an extracellular N-terminal. It has a single TM span and a cytosolic C-terminal tail. Nowadays, it is known that there is an important connection between GPA and band 3 during their biosynthesis and trafficking to plasma membrane [51,52]. In fact, band 3 is critical for GPA synthesis and stability. This GP is tightly associated to protein band 3 in RBC membrane. Accordingly they are present in similar amounts about $10^{6}$ copies per cell. The interaction between these two main integral proteins of RBC membrane originates the Wright $\left(\mathrm{Wr}^{\mathrm{b}}\right)$ antigen [39,51].

Total surface charge density is not affected in GPA-deficient red cells. However, they exhibit increased glycosylation of band 3, probably due to the addition of excessive sialic acid, which should have been present on the GPA protein [52]. These cells maintain a normal RBC shape and deformability, thus suggesting that GPA may not be crucial to the RBC mechanical stability, deformability and shape change.

\subsection{Blood group antigens}

Human beings can be classified into several different blood groups. The ABO blood group system is the mostly used. However there are more, such as Rh blood group, P blood group, Lutheran blood group, Kell blood group, Lewis blood group, Puffy blood group, Kiddy blood group, LW blood group, li blood group and the Diego and Wright blood group antigens on band 3. There are also some other minor blood group antigens such as Chido and the Rodgers blood group systems. These diverse blood group systems comprise about 243 different determinants expressed in RBC membrane [52].

\section{Anchoring and cytoskeleton proteins}

Erythrocyte cytoskeleton is bound to the erythrocyte membrane through high-affinity protein-protein interactions, $[1,3,4,11,17,19,23-25,31,34,35,40,41,50,52,55,56]$. The two major membrane protein responsible for the attachment of the cytoskeleton network to the RBC membrane are band 3 and GPC/D (see Fig. 1). The first one is band 3 macrocomplex in which ankyrin/protein 4.2 are responsible for the interaction with the cytoskeleton, spectrin/actin complex. Ankyrin is an anchoring protein that bridges the tight association between spectrin and band $3[11,24,50]$. The binding can be modulated by the extent of phosphorylation by protein kinase A (PKA), casein kinase I or cyclic AMP-independent protein kinase. Protein 4.2 is also an anchoring protein that interacts with ankyrin and participates in band 3 macrocomplex structure. This protein has also an important role in the interaction between protein band 3 macrocomplex and the Rh complex, by its interactions with CD47 [52].

The second attachment point is composed by GPC/D that can interact with the protein 4.1 complex and as well with the spectrin/actin complex $[1,4,17,24,31,34,35,41,50,52,55]$. There is also a minor site of attachment between cytoskeleton and RBC membrane in which protein 4.1 complex binds to the tetrameric form of band 3 and spectrin/actin (see Fig. 1) [1,19,24,25,34,35].

The three principal "cytoskeleton proteins" are spectrin $(\alpha$ and $\beta)$, actin and protein 4.1. These three proteins form the "junctional complex" [52]. The erythrocyte membrane-cytoskeleton interactions involving this complex can be modulated by phosphorylation of $4.1 \mathrm{R}$ by protein kinase C, PKA, calmodulin or casein kinase. These phosphorylations lead to a decrease of the interactions between 4.1Rspectrin-actin and also 4.1R-Band 3 or 4.1R-GPC/D [19,25]. RBC membrane deformability is highly dependent of these interactions [35].

Importantly, the erythrocyte cytoskeleton and the bilayer membrane are attached not just by proteinprotein interactions, as it was mentioned above, but also by protein-lipid interactions, for instance, by a direct spectrin-lipid interaction mainly with PE $[11,40]$. 


\section{RBC adhesion molecules}

RBCs are generally considered nonadhesive cells. However, several studies have reported the expression of a large number of adhesion molecules in RBC such as CD44, CD47, CD58, LW/ICAM-4, RAGE and $\mathrm{Lu}[5-7,12,16,18,20-22,26,28,36,44-46,48,49,54]$. Many of these adhesion proteins belong to the Immunoglobulin superfamily (IgSF) of proteins [5]. Adhesion molecules play a crucial role in cellcell and cell-tissue interactions. Additionally, they have been implicated in a large range of biological functions such as erythropoiesis (differentiation, maturation, enucleation and release of RBCs), selfrecognition mechanisms, red cell turnover and cell aging $[20,22,46]$. The several adhesion molecules are differentially expressed at distinct stages of the life of RBCs (see Table 2) [20].

Normal RBCs do not adhere to circulating cells and vessel walls under normal circumstances, suggesting that RBC adhesion molecules could be inaccessible to their ligands. However RBCs become more adherent during particular haemostatic conditions (for instance, clot formation), inflammatory process, pathologic occlusion conditions and sickle cell disease [18,20,48,54].

The array of adhesion molecules that will be described following suggest the idea that RBCs have at least the potential capability of adhering to a number of ligands including thrombospondin, fibronectin, laminin, hyaluronan, and thus to other cellular types such as endothelial cells and leukocytes.

\section{1. $L W / I C A M-4$}

LW is also known as intercellular adhesion molecule-4 (ICAM-4) or CD242. This glycoprotein is an erythroid-specific membrane component that may potentially play a role in adhesion or cell interaction events including hemostasis and thrombosis [18,20,22,26,45,46]. ICAM-4 apparently is expressed as part of the Rh macrocomplex in RBC membrane $[45,46]$.

ICAM-4 is a member of the ICAM family. The primary cellular counter-receptor is leukocyte specific $\beta_{2}$ integrins $[10,14]$. However ICAM-4 is an unusual ICAM because it interacts with several types of integrins expressed on blood and endothelium cells $[18,20,22,26]$. This glycoprotein binds to CD11a/CD18 (LFA-1 or $\alpha_{\mathrm{L}} \beta_{2}$ integrin), to CD11b/CD18 (Mac-1 or $\alpha_{\mathrm{M}} \beta_{2}$ integrin), and to $\alpha_{5}$ integrins

Table 2

Adhesion molecules expressed by circulating erythrocyte

\begin{tabular}{|c|c|}
\hline Adhesion molecule and alternate name(s) & Ligand/adhesive function \\
\hline Indian (In(Lu)-related p80, CD44) & Hyaluronan, possibly also fibronectin \\
\hline Rh-related integrin-associated protein (IAP, CD47) & Thrombospondin \\
\hline Lymphocyte-associated antigen-3 (LFA-3, CD58) & $\mathrm{CD} 2$ \\
\hline CD99, MIC2 gene product & $\begin{array}{l}\text { Lymphocyte CD99 is necessary for formation of T-cell } \\
\text { rosettes }\end{array}$ \\
\hline JMH (CD10B, SEMA 7A) & Possible role in adhesion of activated lymphocytes \\
\hline $\mathrm{Ok}^{\mathrm{a}}$ (neurothelin, CD147) & Type IV collagen. Fibronectin, laminin in other tissues \\
\hline LW (ICAM-4, CD242) & $\begin{array}{l}\text { Leukocyte integrins }(\alpha 4 \beta 1, \alpha 4 \beta 3, \alpha \mathrm{v} \beta 1) \text {, platelet integrin } \\
(\alpha \operatorname{Il} \beta 3) \text {, vascular integrin }(\alpha \mathrm{v} \beta 3)\end{array}$ \\
\hline Lutheran (B-CAM/LU, CD239) & Laminin, possibly also integrins \\
\hline Scianna (ERMAP) & Putative adhesive function \\
\hline MER2 (CD151) & Forms laminin-binding complexes with integrins \\
\hline $\begin{array}{l}\text { CD36 (reticulocytes only), platelet glycoprotein IV, } \mathrm{Nak}^{\mathrm{a}} \\
\text { (platelets) }\end{array}$ & Trombospondin (platelets) \\
\hline VLA-4 (reticulocytes only), $\alpha 4 \beta 1$ integrin (CD49d/CD29) & Trombospondin, VCAM-1, fibronectin \\
\hline
\end{tabular}


on nonhemopoietic cells; $\alpha_{4} \beta_{1}$ on hemopoietic cells, and $\alpha_{\mathrm{IIb}} \beta_{3}$ on platelets [2,22,26]. ICAM-4 has also been recently shown as a ligand for monocyte/macrophage-specific CD11c/CD18 [22].

Selective binding of ICAM-4 to different integrins may be important for a variety of normal RBC functions, and also relevant to the pathology of thrombotic events in sickle cell disease.

\section{2. $B-C A M / L U$}

Basal-cell adhesion molecule or Lutheran blood group glycoprotein (B-CAM/LU) was firstly described as a protein expressed at the basal surface of epithelial cells. This protein has a type- 1 transmembrane glycoprotein containing five IgSF domains: two V-types and three C2-types [45,46,48,54].

$\mathrm{B}-\mathrm{CAM} / \mathrm{LU}$ is a laminin- $\alpha 5$ receptor relatively inactive in normal RBC surface. Interestingly, it is highly expressed in sickle RBCs (SS RBCs) surface. The increase of adhesion to lamin in SS RBCs may be connected with the increase expression of B-CAM/LU in these cells $[48,54]$.

\section{3. $C D 47$}

CD47 is a $50 \mathrm{kDa}$ and highly glycosilated plasma membrane. It can also be called as integrinassociated protein (IAP) because its functions have been best studied in relation to integrin signalling. It is a transmembrane glycoprotein with five membrane spanning domains and a single extracellular V-type immunoglobulin super family (IgSF) domain [6].

This glycoprotein is associated with the $\mathrm{Rh}$ macrocomplex and with the cytoskeleton. It is functionally coupled to heterotrimeric Gi proteins, signal regulatory protein $(\operatorname{SIRP} \alpha)$ and cholesterol [7]. CD47 is a member of the thrombospondin (TSP) family member receptor $[5-7,12,45,46]$ and it can interact with several integrins of the $\beta_{1}, \beta_{2}$ and $\beta_{3}$ families modulating platelet activation, cell motility, cell adhesion, leukocyte adhesion, migration and phagocytosis [7]. CD47 cellular functions are associated to the Ig domain that is required for the establishment of interactions with its associated integrins, its ligand TSP and $\operatorname{SIRP} \alpha[5-7,12]$.

In mature RBCs, CD47 has been suggested to mediate cell-cell interaction with SIRP $\alpha$ of splenic macrophages, and to inhibit a phosphorylation cascade that blocks phagocytosis and prevents RBC clearance from the circulation [6].

In SS RBC, the binding of TSP to CD47 generates an intracellular signal that increases SS RBC adhesiveness. This signal is commonly associated to vaso-occlusive events [5,6,12]. CD47 SS RBCs structure is different from the structure of CD47 expressed in normal RBCs. This structural change is responsible for higher ability of SS RBCs to adhere at the endothelium using TSP as a mediator [6].

\subsection{Sialyl moieties}

Sialic acid residues are largely abundant in red cell surface. These residues are mostly present on glycophorins (especially in GPA) but also in band 3 and glycolipids. At physiological pH, sialic acids are negatively charged conferring a halo negative charge to RBCs. This charge usually helps to minimize the interactions between RBCs and also with other blood cells [52]. RBCs aggregation is frequently associated to functions such occlusion of sites of vascular interruption by platelets or as a preliminary stage in the migration of leukocytes out of blood stream and into tissues in the inflammatory process [44,51]. It appears however, that sialic acid residues presented mainly in GPA, could in a certain way help cell-cell interaction of RBCs. In a previous study it was shown that $\mathrm{Le}^{\mathrm{a} / \mathrm{b}}$ antigen present in RBC surface are capable of interacting, even with low affinity, with P-selectin, an integrin that contributes 
to the specificity of interactions among endothelial cells, platelets and leukocytes during inflammation [28]. Matsui et al. have shown a decrease of the adherence of normal and SS RBC to P-selectin, using a P-selective blocking monoclonal antibodies or sialyl Lewis tetrasaccharide. In pre-treating RBCs with sialidase their adherence to activated endothelial cells and immobilized recombinant P-selectin is largely reduced [14]. Additionally sialic acid residues are responsible for the increased adhesion of RBCs to several components of the extracellular matrix, such as laminin and thrombospondin [21].

\section{Disorders on red blood cell membrane}

In clinical haematology, RBC shape abnormalities are still widely used for first step-diagnoses before further detailed characterization of the phenotypes and genotypes of RBC membrane disorders. Interestingly RBC membrane disorders can be regarded either as: (1) abnormalities in vertical interactions of membrane components, or (2) those in their horizontal interactions [52].

An abnormality in vertical interactions occurs mainly in spectrin-ankyrin-band 3 interactions, the protein 4.1-GPC linkage and also at the level of the interaction of skeletal proteins with membrane lipids (PE and PS mainly) [52]. The abnormalities in horizontal interactions usually concern either the selfassociation of spectrin to form its tetramers, which are the most important component of the cytoskeleton network or its interactions to protein 4.1 and/or actin [52].

It has been observed that several of the RBC disorders are tightly associated with mutations occurring in genes codifying for different RBC membrane proteins (see Table 1). For instance, Hereditary Spherocytosis (HS), Hereditary Elliptocytosis (HE) and Southeast Asian Ovalocytosis (SAO) are associated to mutations in peripheral proteins [10,13-15,29,52]. A deficiency of spectrin occurs in HE and HS, while B3 gene mutations are associated with SAO and also HS [10,13-15,29,52]. In Europe about 30\% of the cases of HE has a deficiency in protein 4.1 levels in RBC membrane.

A deficiency in a protein can lead to a secondary reduction of another one. This is the case for instance of a rare type of HS. In this disease the reduction or absence of protein 4.2 is associated to a secondary decrease of CD47 levels, which affects the interaction of B3 macrocomplex with Rh complex $[13,15,53]$.

Lipid disorders, with a hereditary origin, are apparently rarer than the protein ones. Interestingly, Japanese people seem to be more affected by these disorders than occidental nations. One explanation to this concern the different screening systems used to detect abnormalities of RBC membrane components in different countries or even institutions [52]. The RBC membrane lipid anomalies can be due to a strong abnormal membrane lipid composition, like in hereditary high red cell membrane phosphatidylcholine haemolytic anemia (HPCHA), or to abnormalities in plasma factors such lipoproteins and enzymes, which are observed in $\alpha$ and $\beta$ lipoprotein deficiency or in lecithin: cholesterol acyltransferase deficiency.

\section{Conclusions}

Red blood cells are the main cells in circulation. As it was reviewed in this article, erythrocytes have a very particular membrane structure and composition that supports their features and functions. It has been recently shown that RBCs have the ability to express adhesion molecules on their membrane. These studies may actually open new horizons for the study of the biological functions of erythrocytes in circulation. 


\section{Acknowledgements}

We thank Ângelo Calado (Medical School of University of Lisbon, Portugal) for his carefully reading of the manuscript and also to Fundação para a Ciência e Tecnologia (FCT) for the financial support (PTDC/SAU-OSM/73449/2006).

\section{References}

[1] X. An, Y. Takakuwa, W. Nunomura, S. Manno and N. Mohandas, Modulation of band 3-ankyrin interaction by protein 4.1, The Journal of Biological Chemistry 271 (1996), 33187-33191.

[2] N. Babu, Influence of hypercholesterolemia on deformability and shape parameters of erythrocytes in hyperglycemic subjects, Clinical Hemorheology and Microcirculation 41(3) (2009), 169-177.

[3] L. Bordin, A.M. Brunati, A. Donella-Deana, B. Baggio, A. Toninello and G. Clari, Band 3 is an anchor protein and a target for SHP-2 tyrosine phosphatase in human erythrocytes, Blood 100 (2002), 276-282.

[4] L. Bordin, F. Zen, F. Ion-Popa, M. Barbetta, B. Baggio and G. Clari, Band 3 tyr-phosphorylation in normal and glucose6-phosphate dehydrogenase-deficient human erythrocytes, Molecular Membrane Biology 22(5) (2005), 411-420.

[5] J.E. Brittain, J. Hans, K.I. Ataga, E.P. Orringer and L.V. Paris, Mechanism of CD47-induced $\alpha_{4} \beta_{1}$ integrin activation and adhesion in sickle reticulocytes, The Journal of Biological Chemistry 279(41) (2004), 42393-42402.

[6] J.E. Brittain, K.J. Mlinar, C.S. Anderson, E.P. Orringer and L.V. Paris, Integrin-associated protein is an adhesion receptor on sickle red blood cells for immobilized thrombospondin, Blood 97(7) (2001), 2159-2164.

[7] E.J. Brown and W.A. Frazier, Integrin-associated Protein (CD47) and its ligands, Trends in Cell Biology 11(3) (2001), $130-135$.

[8] A.M. Brunati, L. Bordin, G. Clari, P. James, M. Quadroni, E. Bariotono, L.A. Pinna and A. Donella-Deana, Sequential phosphorilation of protein band 3 by Syk and Lyn tyrosine kinases in intact human erythrocytes: identification of primary and secondary phosphorylation sites, Blood 96 (2000), 1550-1557.

[9] M.E. Campanella, H. Chu and P.S. Low, Assembly and regulation of a glycolytic enzyme complex on the human erythrocyte membrane, Proceedings of National Academic Sciences 102 (2005), 2402-2407.

[10] J.C. Cheung and R.A. Reithmeier, Membrane integration and topology of the first transmembrane segment in normal and Southeast Asian ovalocytosis human erythrocyte anion exchanger 1, Molecular Membrane Biology 22(3) (2005), 203-214.

[11] A. Czogalla, A.R. Jaszewski, W. Diakowski, E. Bok, A. Jezierski and A.F. Sikorski, Structural insight into an ankyrinsensitive lipid-binding site of erythroid $\beta$-spectrin, Molecular Membrane Biology 24(3) (2007), 215-224.

[12] K.D. Dahl, C.M. Westhoff and D.E. Discher, Fractional attachment of CD47 (IAP) to the erythrocyte cytoskeleton and visual colocalization with Rh protein complexes, Blood 101(3) (2003), 1194-1199.

[13] J. Delaunay, The molecular basis of hereditary red cell membrane disorders, Blood Reviews 21 (2006), 1-20.

[14] D. Dhermy, O. Burnier, M. Bougeois and B. Grandchamp, The red blood cell band 3 variant (band 3 Biceêtrel: R490C) associated with dominant hereditary spherocytosis causes defective membrane targeting of the molecule and a dominant negative effect, Molecular Membrane Biology 16(4) (1999), 305-312.

[15] P.G. Gallagher, Red cell membrane disorder, Hematology, Eduacation Program, American Society of Hematology, 2005, 13-18.

[16] G. Garratty, M.J. Telen and L.D. Petz, Red cell antigens as functional molecules and obstacles to transfusion, Hematology (2002), 445-462.

[17] J.A. Gimm, X. An, W. Nunomura and N. Mohandas, Functional characterization of spectrin-actin-binding domains in 4.1 family of proteins, Biochemistry 41 (2002), 7275-7282.

[18] M.S. Goel and S.L. Diamond, Adhesion of normal erythrocytes at depresses venous shear rates to activated neuthrophils, activated platelets, and fibrin polymerized from plasma, Blood 100(10) (2002), 3797-3803.

[19] B.-G. Han, W. Nunomura, Y. Takakuwa, N. Mohandas and B.K. Jap, Protein 4.1R core domain structure and insights into regulation of cytoskeletal organization, Natural Structural and Molecular Biology 7 (2000), 871-875.

[20] P. Hermand, P. Gane, M. Huet, V. Jallus, C. Kaplans, H.H. Sonneborn, J.-P. Cartron and P. Bailly, Red cell ICAM-4 is a novel ligand for platelets-activated $\alpha_{I I b} \beta_{3}$ integrin, The Journal of Biological Chemistry 278(7) (2002), 4892-4898.

[21] C.A. Hillery, M.C. Du, R.R. Montgomery and J.P. Scott, Increased adhesion of erythrocytes to componentes of the extracellular matrix: isolation and characterization of a red blood cell lipid that binds thrombospondin and laminin, Blood 87(11) (1996), 4879-4886.

[22] E. Ihanus, L.M. Uotila, A. Toivanen, M. Varis and C.G. Gahmberg, Red-cell ICAM4 is a ligand for the monocyte/macrophage integrin CD11c/CD18: characterization of the binding sites on ICAM-4, Blood 109(2) (2007), 802-810. 
[23] N.G. de Isla, B.D. Riquelma, R.J. Rasia, J.R. Valverde and J.F. Stoltz, Quantification of glycophorin A and glycophorin B on normal human RBCs by flow cytometry, Transfusion 43 (2003), 1145-1152.

[24] P.S. Low, B.M. Willardson; N. Mohandas, M. Rossi and S. Shohet, Contribution of the band 3-ankyrin interaction to erythrocyte membrane mechanical stability, Blood 77 (1991), 1581-1586.

[25] S. Mannot, Y. Takakuwa and N. Mohandas, Modulation of erythrocyte membrane mechanical function by protein 4.1 phosphorylation, The Journal of Biological Chemistry 280 (2005), 7581-7587.

[26] T.J. Mankelow, F.A. Sping, S.F. Parsons, R.L. Brady, N. Mohandas, J.A. Chasis and D.J. Anstee, Identification of critical amino-acid residues on the erythroid intercellular adhesion molecule-4 (ICAM-4) mediating adhesion to $\alpha_{V}$ integrin, Blood 103(4) (2004), 1503-1508.

[27] A. Marossy, P. Svorc, I. Kron and S. Gresová, Hemorheology and circulation, Clinical Hemorheology and Microcirculation 42(4) (2009), 239-258.

[28] N.M. Matsui, L. Borsig, S.D. Rosen, M. Yaghmai, A. Varki and S.H. Embury, P-selectin mediates the adhesion of sickle erythrocytes to the endothelium, Blood 98(6) (2001), 1955-1962.

[29] M.F. McMullin, The molecular basis of disorders of the red cell membrane, Journal of Clinical Pathology 32 (1999), $245-248$.

[30] M. Merten, C. Beythien, K. Gutensohn, P. Kühnl, T. Meinertz and P. Thiagarajan, Sulfatides activate platelets through Pselectin and enhance platelet and platelet-leukocyte aggregation, Arteriosclerosis Thrombosis Vascular Biology 25 (2005), 258-263.

[31] R. Moriyama, C.R. Lombardo, R.F. Workman and P.S. Low, Regulation of linkages between the erythrocyte membrane and its skeleton by 2,3-DPG, The Journal of Biological Chemistry 268 (1993), 10990-10996.

[32] S.C. Murphy, N.L. Hiller, T. Harrison, J.W. Lomasney, N. Mohandas and K. Haldar, Lipid rafts and malaria parasite infection of erythrocytes, Molecular Membrane Biology 23(1) (2006), 81-88.

[33] G.B. Nash, T. Watts, C. Thornton and M. Barigou, Red cell aggregation as a factor of influencing margination and adhesion of leukocytes and platelets, Clinical Hemorheology and Microcirculation 39(1-4) (2008), 303-310.

[34] V. Nicolas, I. Mouro-Chanteloup, C. Lopez, P. Gane, A. Gimm, N. Mohandas, J.-P. Cartron, C. Le Van Kim and Y. Colin, Functional interaction between Rh proteins and the spectrin-based skeleton in erythroid and epithelial cells, Transfusions Clinique et Biologique 13 (2006), 23-28.

[35] S. de Oliveira, A.S. Silva-Herdade and C. Saldanha, Modulation of erythrocyte deformability by PKC activity, Clinical Hemorheology and Microcirculation 39(1-4) (2008), 363-373.

[36] E.M. Pasini, M. Kirkegaard, P. Mortensen, H.U. Lutz, A.W. Thomas and M. Mann, In-depth analysis of the membrane and cytosolic proteome of red blood cells, Blood 108 (2006), 791-801.

[37] L.J. Pike, Lipid rafts: bringing order to chaos, Journal of Lipid Research 44 (2003), 655-667.

[38] R. Plasenzotti, U. Windberger, F. Ulberth, W. Osterode and U. Losert, Influence of fatty acid composition in mammalian erythrocytes on cellular aggregation, Clinical Hemorheology and Microcirculation 37(3) (2007), 237-243.

[39] J. Poole, Red cell antigens on band 3 and glycophorin A, Blood Reviews 14 (2000), 31-43.

[40] S. Ray and A. Chakrabarti, Membrane interaction of erythroid spectrin: surface-density-dependent high-affinity binding to phosphatidylethanolamine, Molecular Membrane Biology 21 (2004), 93-100.

[41] C. Saldanha, A.S. Silva, S. Gonçalves and J. Martins-Silva, Modulation of erythrocyte hemorheological properties by band 3 phosphorylation and dephosphorylation, Clinical Hemorheology and Microcirculation 36(3) (2007), $183-194$.

[42] U. Salzer and R. Prohaska, Stomatin, flotillin-1 and flotillin-2 are major integral proteins of erythrocyte lipid rafts, Blood 97(4) (2001), 1141-1143.

[43] B.U. Samuel, N. Mohandas, T. Harrison, H. McManus, W. Rosse, M. Reid and K. Haldar, The role of cholesterol and glycosylphosphatidylinositol-anchored proteins of erythrocyte rafts in regulating raft protein content and malaria infection, The Journal of Biological Chemistry 276(31) (2001), 29319-29329.

[44] L. Sargento, C. Saldanha, J. Monteiro, C. Perdigão and J. Martins-Silva, Evidence of prolonged disturbances in the haemostatic, hemorheologic and inflammatory profiles in transmural myocardial infarction survivors, Thrombosis and Haemostasis 89(5) (2003), 892-903.

[45] M.J. Telen, Red blood cell surface adhesion molecules: their possible roles in normal human physiology and disease, Seminars in Hematology 37(2) (2000), 130-142.

[46] M.J. Telen, Erythrocyte adhesion receptors: blood group antigens and related molecules, Transfusion Medicine Reviews 19(1) (2005), 32-44

[47] M. Uyuklu, H.J. Meiselman and O.K. Baskurt, Effect of hemoglobin oxygenation level on red blood cell deformability and aggregation parameters, Clinical Hemorheology and Microcirculation 41(3) (2009), 179-188.

[48] J.L. Wautier and M.P. Wautier, Erythrocytes and platelet adhesion to endothelium are mediated by specialized molecules, Clinical Hemorheology and Microcirculation 30(3,4) (2004), 181-184.

[49] M.P. Wautier, T. Khodabandehlou, C. Le Dévéhat and J.L. Wautier, Modulation of RAGE expression influences the adhesion of red blood cells from diabetic patient, Clinical Hemorheology and Microcirulation 35(3) (2006), 379-386. 
[50] B.M. Willardson, B.J.M. Thevenin, M.L. Harrison, W.M. Kuster, M.D. Benson and P.S. Low, Localization of the ankyrinbinding site on erythrocyte membrane protein band 3, The Journal of Biological Chemistry 264 (1989), 15893-15899.

[51] R.C. Williamson and A.M. Toye, Glycophorin A: band 3 aid, Blood Cells, Molecules and Diseases (2008), 1-9.

[52] Y. Yawata, Cell Membrane: The Red Blood Cell as a Model, Wiley, 2003.

[53] D. Zang, A. Kiyatkin, J.T. Bolin and P.S. Low, Crystallographic structure and functional interpretation of the cytoplasmic domain of erythrocyte membrane band 3, Blood 96 (2000), 2925-2933.

[54] Q. Zen, M. Cottman, G. Truskey, R. Fraser and M.J. Telen, Critical factors in basal cell adhesion molecule/Lutheranmediated adhesion to laminin, The Journal of Biological Chemistry 274(2) (1999), 728-734.

[55] Y. Zhang, L.R. Manning, J. Falcones, O. Platt and J.M. Manning, Human erythrocyte membrane band 3 protein influences hemoglobin cooperativity, The Journal of Biological Chemistry 278 (2003), 39565-39571.

[56] Y. Zipser, A. Piade, A. Barbul, R. Korenstein and N.S. Kosower, $\mathrm{Ca}^{2+}$ promotes erythrocyte band 3 tyrosine phosphorylation via dissociation of phosphotyrosine phosphatase from band 3, Biochemistry Journal 368 (2002), 137-144. 